\title{
A FORMAL SERVICE SPECIFICATION FOR IIOP BASED ON ISO/IEC 14752
}

\author{
Abhishek Singh and Jonathan Billington \\ 1.IBM Global Services Australia 2. Computer Systems Engineering Centre, University of South \\ Australia
}

\begin{abstract}
The Internet Inter-ORB Protocol (IIOP) supports the interworking of Object Request Brokers (ORBs) over TCP/IP. This paper creates a service specification for IIOP by combining three interworking facilities identified in ISO/IEC 14752. This is formalised using Coloured Petri nets, which are used to generate the set of global primitive sequences of the IIOP service.
\end{abstract}

Keywords IIOP, CORBA, ODP, Coloured Petri Nets, Service Specification

\section{Introduction}

The Common Object Request Broker Architecture (CORBA) (Object Management Group, 2001), has been proposed by the Object Management Group (OMG) (see http://www.omg.org) as an open standard for Object Oriented Middleware (International Systems Group, 2001). CORBA achieves interoperability between heterogeneous Object Request Brokers (ORBs) via its Internet Inter-ORB Protocol (IIOP) (see http://www.omg.org) recently adopted as an international standard, ISO/IEC 19500-2 (ISO/IEC 19500-2, 2000). IIOP provides a reliable request/response service for object interoperability over TCP/IP, making it suitable for interoperability between Web-based applications. IIOP operates in a seamless way, ensuring that implementation and location differences are transparent to the interoperating ORBs.

The OMG specification (Object Management Group, 2001) has not defined the services of IIOP explicitly. ISO/IEC 14752 (ITU X.931, 1999), a standard for Open Distributed Processing (ODP) (ITU X.903, 1995), defines how interactions between objects in a computational specification of a 
system relate to protocol support for those interactions via a General Interworking Framework (GIF). The GIF comprises a set of facilities, each consisting of a set of functionally-related service primitives (ITU X.210, 1993). The standard (ITU X.931, 1999) defines the parameters of the service primitives and the permitted sequences of the service primitives at a local interface by means of state tables. The standard is limited because sequences are only defined within a particular facility and the ordering relationships between primitive occurrences at the different ORBs are not defined. There is no attempt to define the combined set of primitive sequences when multiple facilities are composed.

This paper defines the local sequencing of the service primitives belonging to the three GIF facilities supported by IIOP and presents them in state tables. The global sequences of service primitives are then generated from a Coloured Petri Net (Jensen, 1997) model of the service, based on the state tables and assumptions concerning the behaviour of IIOP. Coloured Petri Nets are suitable for specifying services $[8,10]$ and have previously been used in other protocol applications [11,12].

\section{Using ISO/IEC 14752 for Specifying the Service of IIOP}

The General Interworking Framework (GIF) comprises a set of four facilities. The basic interworking facility defines primitives that directly support computational interactions. The location facility allows a client to request a location-related service from the server. The access facility allows the client and server to negotiate the abstract and transfer syntax to be used for the transmission of data and the association management facility is concerned with connection management. Each facility supports the liaison between a protocol service provider and its users, by defining a set of service primitives.

IIOP supported GIF facilities are identified in Annex A of the ISO/IEC 14752 (ITU X.931, 1999). The three facilities supported are the basic interworking facility, the location facility and the association management facility.

\subsection{Defining Service Primitives}

Service primitives are abstractions of layer-to-layer communication in a protocol stack. In IIOP, services are provided to client and server ORBs. Table 1 presents the GIF facilities supported by IIOP and their meaning. GIF primitives may be either of type submit or deliver. A user issues submit 
primitives while the service provider issues deliver primitives. The standard also identifies the parameters associated with each GIF primitive. More detail on IIOP service parameters can be found in CORBA's specification (Object Management Group, 2001) and ISO/IEC 14752 (ITU X.931, 1999).

Table 1. GIF Facilities used by the IIOP Service

\begin{tabular}{|c|c|c|}
\hline GIF Facility & GIF primitive & Meaning \\
\hline \multirow{5}{*}{$\begin{array}{l}\text { Association } \\
\text { Management } \\
\text { Facility }\end{array}$} & Association-request \{submit, deliver & $\begin{array}{l}\text { Client sends a connection request } \\
\text { to the server }\end{array}$ \\
\hline & Association-accept $\{$ submit, deliver $\}$ & $\begin{array}{l}\text { Server accepts the connection } \\
\text { request }\end{array}$ \\
\hline & Association-reject $\{$ submit, deliver $\}$ & $\begin{array}{l}\text { Server rejects the connection } \\
\text { request from the client }\end{array}$ \\
\hline & Association-close \{submit, deliver\} & $\begin{array}{l}\text { Client informs the server that the } \\
\text { connection is closed }\end{array}$ \\
\hline & Association-abort \{submit, deliver\} & $\begin{array}{l}\text { Server informs the client that the } \\
\text { connection is closed or failure in } \\
\text { the underlying communication } \\
\text { layer has occurred }\end{array}$ \\
\hline \multirow{4}{*}{$\begin{array}{l}\text { Basic } \\
\text { Interworking } \\
\text { Facility }\end{array}$} & Request \{submit, deliver\} & Client requests a service \\
\hline & Result $\{$ submit, deliver $\}$ & $\begin{array}{l}\text { A result is returned by the server } \\
\text { to the client to satisfy the request }\end{array}$ \\
\hline & Cancel $\{$ submit, deliver $\}$ & $\begin{array}{l}\text { Client requests cancellation of the } \\
\text { previously requested service }\end{array}$ \\
\hline & Abort deliver & $\begin{array}{l}\text { Client and server are informed of } \\
\text { a failure in the underlying layer }\end{array}$ \\
\hline \multirow[t]{4}{*}{$\begin{array}{l}\text { Location } \\
\text { Facility }\end{array}$} & Location-query \{submit, deliver\} & $\begin{array}{l}\text { Client requests a location related } \\
\text { service from the server }\end{array}$ \\
\hline & Location-advise \{submit, deliver\} & $\begin{array}{l}\text { Location related advice is } \\
\text { returned to to satisfy the query }\end{array}$ \\
\hline & Location-cancel \{submit, deliver\} & $\begin{array}{l}\text { Client requests cancellation of a } \\
\text { location related service }\end{array}$ \\
\hline & Location-abort deliver & $\begin{array}{l}\text { Client and server are informed of } \\
\text { a failure in the underlying layer }\end{array}$ \\
\hline
\end{tabular}

\subsection{Local Sequences of Service Primitives}

This section specifies the sequencing of the service primitives when the 3 facilities are combined. This sequencing is not included in the standard (ITU X.931, 1999). The constraints on the sequencing of the service primitives are determined after an analysis of the CORBA specification (Object Management Group, 2001). We define the (local) sequences using state tables for each of the IIOP client and server.

The state tables are derived by combining the state tables for each facility supported by IIOP and by applying possible constraints specified in the 
CORBA specification. Table 2 and Table 3 are the state tables for the IIOP client and server respectively.

The service primitives for the client and server-side ORBs are asymmetric. Hence, for each side, a separate state machine exists for each request reference. A request reference uniquely identifies a request that can be used by subsequent primitives to unambiguously refer to this request among various request primitives.

Table 2. State Table for IIOP Client

\begin{tabular}{|c|c|c|c|c|c|c|}
\hline $\begin{array}{l}\text { State } \\
\text { Event }\end{array}$ & idle & $\begin{array}{l}\text { assn_req } \\
\text { pend }\end{array}$ & established & wait_reply & querying & $\begin{array}{l}\text { wait_reply } \\
\text { querying }\end{array}$ \\
\hline $\begin{array}{l}\text { association- } \\
\text { request } \\
\text { submit }\end{array}$ & $\begin{array}{l}\text { assn } \\
\text { req } \bar{p} \\
\text { end }\end{array}$ & & & & & \\
\hline $\begin{array}{l}\text { association- } \\
\text { accept } \\
\text { deliver }\end{array}$ & & $\begin{array}{l}\text { establish } \\
\text { ed }\end{array}$ & & & & \\
\hline $\begin{array}{l}\text { association- } \\
\text { reject } \\
\text { deliver }\end{array}$ & & idle & & & & \\
\hline $\begin{array}{l}\text { request } \\
\text { submit }\end{array}$ & & & wait_reply & & $\begin{array}{l}\text { wait_reply_ } \\
\text { querying }\end{array}$ & \\
\hline $\begin{array}{l}\text { result } \\
\text { deliver }\end{array}$ & & & & established & & querying \\
\hline $\begin{array}{l}\text { cancel } \\
\text { submit }\end{array}$ & & & & established & & querying \\
\hline $\begin{array}{l}\text { location- } \\
\text { query } \\
\text { submit }\end{array}$ & & & querying & $\begin{array}{l}\text { wait_reply_ } \\
\text { querying }\end{array}$ & & \\
\hline $\begin{array}{l}\text { location- } \\
\text { advise } \\
\text { deliver }\end{array}$ & & & & & established & wait_reply \\
\hline $\begin{array}{l}\text { location- } \\
\text { cancel } \\
\text { submit }\end{array}$ & & & & & established & wait_reply \\
\hline $\begin{array}{l}\text { association- } \\
\text { abort } \\
\text { submit }\end{array}$ & & idle & idle & idle & idle & idle \\
\hline $\begin{array}{l}\text { association- } \\
\text { close } \\
\text { deliver }\end{array}$ & & idle & idle & idle & idle & idle \\
\hline $\begin{array}{l}\text { abort } \\
\text { deliver }\end{array}$ & & idle & idle & idle & idle & idle \\
\hline
\end{tabular}

The association management facility defines primitives for the establishment of connections. The basic interworking facility primitives and location facility primitives can only occur after the association management 
facility has established a connection (i.e., the client and the server are in their established states). We therefore use the states from the association management facility state tables (idle, assn_req_pend, assn_ind_pend, established) directly in Tables 2 and 3. Three more states are used, one from the basic interworking facility, when a request is sent by the client and is received by the server (wait_reply) and two from the location facility, when a location query is sent by the client and received by the server (querying, queried).

Table 3. State Table for IIOP Server

\begin{tabular}{|c|c|c|c|c|c|c|}
\hline $\begin{array}{l}\text { State } \\
\text { Event }\end{array}$ & idle & $\begin{array}{l}\text { assn_in } \\
\text { d_pend }\end{array}$ & established & wait_reply & queried & $\begin{array}{l}\text { wait_reply } \\
\text { queried }\end{array}$ \\
\hline $\begin{array}{l}\text { Association } \\
\text {-request } \\
\text { deliver }\end{array}$ & $\begin{array}{l}\text { assn_i } \\
\text { nd_pe } \\
\text { nd }\end{array}$ & & & & & \\
\hline $\begin{array}{l}\text { Association } \\
\text {-accept } \\
\text { submit }\end{array}$ & & $\begin{array}{l}\text { establis } \\
\text { hed }\end{array}$ & & & & \\
\hline $\begin{array}{l}\text { Association } \\
\text {-reject } \\
\text { submit } \\
\end{array}$ & & idle & & & & \\
\hline $\begin{array}{l}\text { request } \\
\text { deliver }\end{array}$ & & & wait_reply & & $\begin{array}{l}\text { wait_reply_ } \\
\text { queried }\end{array}$ & \\
\hline $\begin{array}{l}\text { result } \\
\text { submit }\end{array}$ & & & & established & & queried \\
\hline $\begin{array}{l}\text { cancel } \\
\text { deliver }\end{array}$ & & & & established & & queried \\
\hline $\begin{array}{l}\text { location- } \\
\text { query } \\
\text { deliver }\end{array}$ & & & queried & $\begin{array}{l}\text { wait_reply } \\
\text { queried }\end{array}$ & & \\
\hline $\begin{array}{l}\text { location- } \\
\text { advise } \\
\text { submit }\end{array}$ & & & & & established & wait_reply \\
\hline $\begin{array}{l}\text { location- } \\
\text { cancel } \\
\text { deliver }\end{array}$ & & & & & established & wait_reply \\
\hline $\begin{array}{l}\text { Association } \\
\text {-abort } \\
\text { deliver } \\
\end{array}$ & & idle & idle & idle & idle & idle \\
\hline $\begin{array}{l}\text { Association } \\
\text {-close } \\
\text { submit } \\
\end{array}$ & & idle & idle & & & \\
\hline $\begin{array}{l}\text { abort } \\
\text { deliver }\end{array}$ & & idle & idle & idle & idle & idle \\
\hline
\end{tabular}

The client and server can issue basic interworking facility and location facility primitives and can have outstanding requests from these two 
facilities, hence two composite states are introduced (wait_reply_querying, wait_reply_queried). Given that any number of basic interworking facility and location facility primitives can occur over a single connection, requests are associated with unique request identifiers to differentiate them from all other request primitives. The abort.deliver of the basic interworking facility and the location-abort.deliver of the location facility are combined into a single primitive, because they represent the same operation of informing the client and the server that failure in the underlying communication layer has occurred. Hence, a single abort deliver primitive is used in the state tables.

\section{Colored Petri Nets}

Coloured Petri Nets (Jensen, 1997) (CPNs) were developed in the early 1980 's by Kurt Jensen at Aarhus University in Denmark. CPNs are a widely used technique mainly because of their graphical representation and a welldefined semantics which is necessary for formal analysis (Kristensen et al., 1998).

The CPNs presented in this paper were edited, simulated and analysed using a software support tool called Design/CPN (Kristensen et al., 1998). Hierarchical constructs allow CPNs to be organised in a modular way. Separate pages are used to draw individual nets to manage model complexity and increase readability of the net. The CPNs presented in this paper rely on a construct known as fusion places, which is supported in Design/CPN. Fusion places allow places to be duplicated in various CPN pages but to be considered logically identical. Design/CPN has a simulator, which supports execution of CPN models. Design/CPN performs state space analysis, generating an occurrence graph (OG) from the CPN. The OG can be analysed to determine dynamic properties of the CPN (e.g., deadlocks, livelock). We use Design/CPN to generate the OG of the IIOP service, and then import it to a finite state automata tool, FSM (AT\&T Research Labs, FSM), to generate the minimised deterministic service language automaton.

\section{CPN Model of IIOP Services}

The purpose of the CPN model of the IIOP service is to generate global sequences of service primitives, as the order of service primitives at the different end points (ORBs) are not given in ISO/IEC 14752. 


\subsection{Modelling Assumptions}

We model communication between the Client (the Object Requestor) and the Server (the Responder) by two separate channels. Each channel represents a uni-directional flow of messages (e.g. one from client to server and the other from server to client). Each channel is assumed to deliver messages in sequence with no loss or duplication. It is reasonable to use a FIFO queue for each channel, as it closely resembles the underlying TCP layer, and IIOP does not introduce any defects.

We assume that we only need to model a single connection instance. The state 'finish' is used in the CPN model, instead of state 'idle' when association-close, association-abort and abort primitives occur. This prevents any further connections being established.

\subsection{CPN Model}

The CPN model of the IIOP Service comprises 9 pages shown in Figure 1 to Figure 9. Figure 1 defines the data for the rest of the figures.

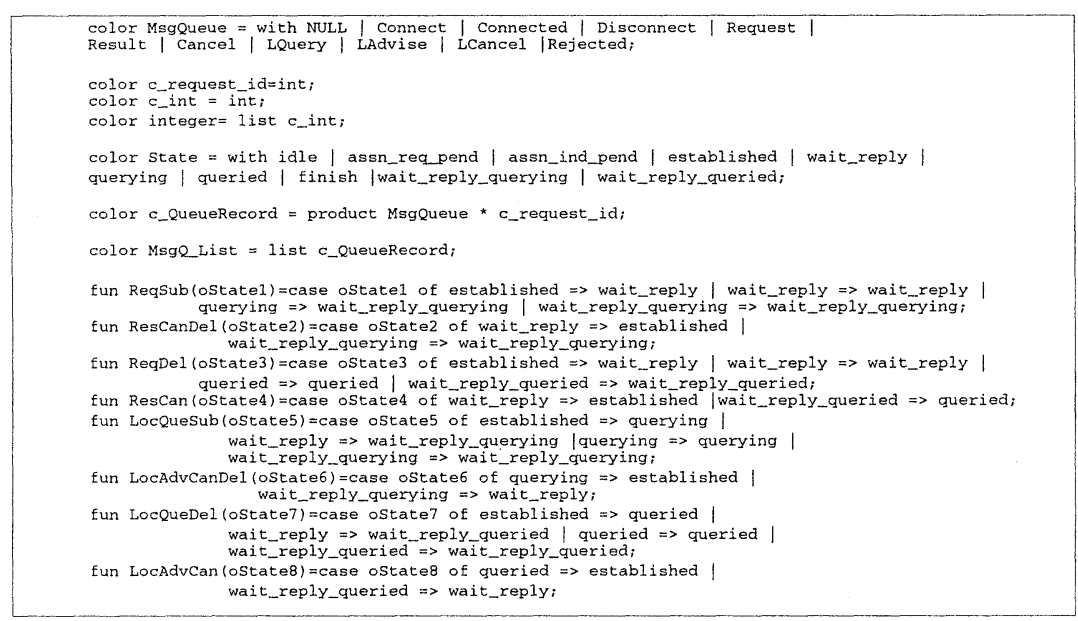

Figure 1. CPN model Declarations

Each of the pages 2 to 9 has a similar structure:

- On the left of each diagram is the client side, while on the right is the server.

- Two fusion places, called Client and Server, model the states of the interface between the requesting and responding ORBs. These places are typed by the color set State.

- The two communication channels are modelled by a further two fusion places, called $\mathrm{CtoS}$ and $\mathrm{StoC}$, shown in the middle of each diagram. 
CtoS stores messages from the Client to the Server and StoC messages from the Server to the Client. These places are typed by the colour set MsgQ_List, which builds a FIFO queue of messages.

- The primitives are represented by transitions, and their occurrence by the firing of the transition.

- The primitives are prefixed with the letter 'I' to indicate that they are IIOP primitives, and they label the corresponding CPN transition.

The CPN model uses various functions and variables declared in the Figure 1. The functions (ReqSub, ReqCanDel, ReqDel, ResCan, LocQueSub, LocAdvCanDel, LocQueDel, LocAdvCan) accepts one of the variables oState(1..8) as its input parameter. The functions control the states of the client and server based on the occurrences of the primitives. The variables oState( $0 . .8)$, vState, vState1 and vState 2 are of type State. The variables (vInt, vInt2, vInt3, inRid, iResRid, oCRid, iCRid, iAdvRids, iLCRid) are of type integer.

There is five more fusion places (Rids, CancelRids, ResultRids, LocCancelRids, AdviseRids) of type State. These places are used to keep track of various Request Ids (Rids) used in the model. An Rid uniquely identifies a request and hence allows a request to be unambiguously referred to by various request primitives.

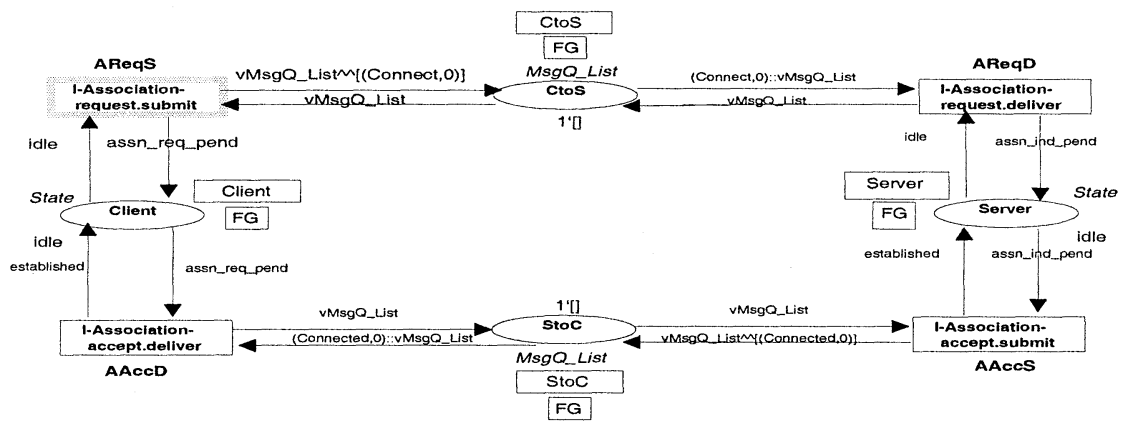

Figure 2. Connection Management CPN

Figure 2 models the sequence of I-Association-request and IAssociation-accept primitives. The initial marking of both Client and Server is idle. The two queues CtoS and StoC have an initial marking of Null, as both buffers are empty. In this marking the only transition that can occur is IAssociation-request.submit, this puts the client into the state assn_req pend. Next, the I-Association-request.deliver can occur followed by the IAssociation-accept.submit transition. Connection establishment is completed on the occurrence of the I-Association-accept.deliver primitive. The client 
and the server are in the state established. If the server rejects the connection request, the I-Association-reject primitives occur as in Figure 3 putting the client and server in idle state.

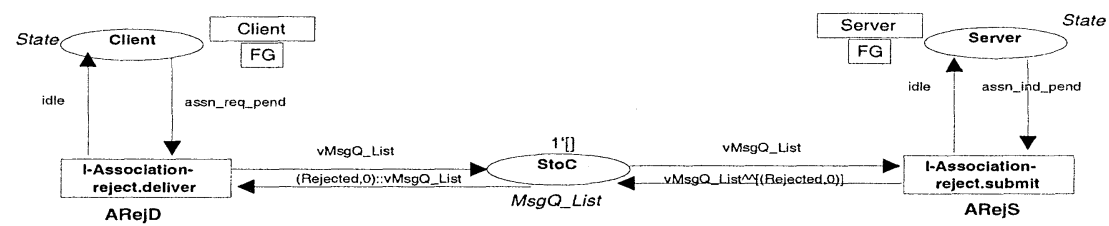

Figure 3. Association Reject CPN

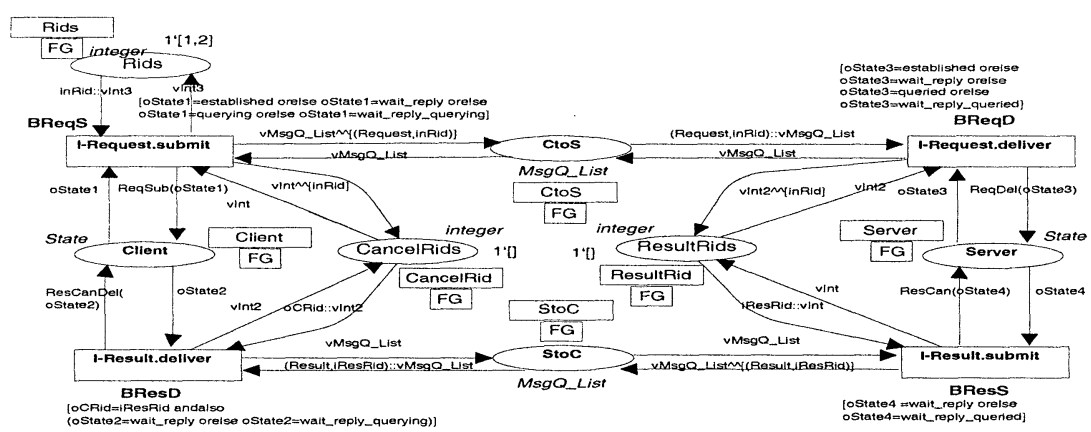

Figure 4. Request \& Result primitives CPN

Figure 4 models the sequence of I-Request and I-Result primitives. IRequest.submit can occur if the client is in one of the states (established, wait_reply, querying, wait_reply_querying) specified in the guard of the transition and there is an Rid available for the request. When IRequest.submit occurs the Rid associated with it is stored in the place CancelRids. The Rid(s) stored in CancelRids is used by the client to associate it with a request cancellation primitive or to keep track of the outstanding request. The I-Request-deliver primitive can occur next given that the server is in one of the states specified by the guard. The server stores Rid in ResultRids to keep track of outstanding Rids. I-Result.submit occurs next, provided there is a Rid in ResultRids. I-Result.deliver can occur next removing the matching Rid from the place CancelRids.

Figure 5 models the sequence of I-Cancel primitive. The first primitive that can occur in this figure is I-Cancel.submit, given the client is in one of the states (wait_reply, wait_reply_querying) specified in the guard and the place CancelRids has a Rid (i.e., a request is outstanding). The transition ICancel.deliver can occur next changing server's state to (established or 
queried) depending on its initial state (i.e., if server is in state wait_reply it is put into state established when I-Cancel.deliver primitive occurs).

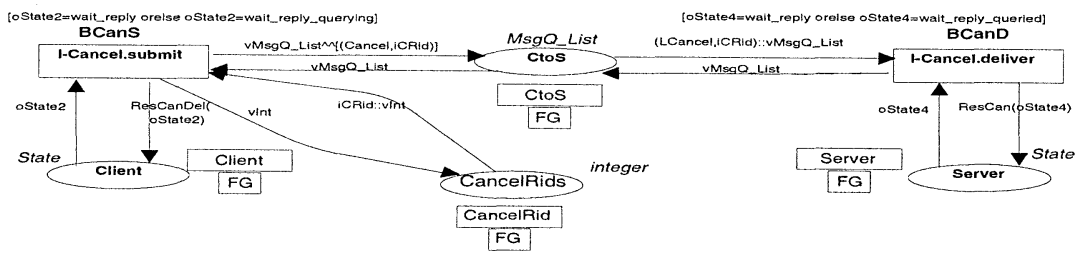

Figure 5. Cancel primitives CPN

Figure 6 models the sequence of I-Location-query and I-Location-advise primitives. I-Location-query.submit can occur if the client is in one of the states (established, wait_reply, querying, wait_reply_querying) specified in the guard of the transition and there is an available Rid to associate with the request in place Rids. When I- Location-query.submit occurs the Rid associated with it is stored in the place LocCancelRids. The Rid(s) stored in LocCancelRids is used by the client to associate it with a location query cancellation primitive or to keep track of the completion of a location query. I-Location-query.deliver occurs next provided the server is in one of the states specified by the guard. The server stores Rid in AdviseRids to keep track of outstanding Rids. I-Location-advise.submit can occur next, provided there is a Rid in AdviseRids. I-Location-advise.deliver can occur next removing the matching Rid from the place LocCancelRids.

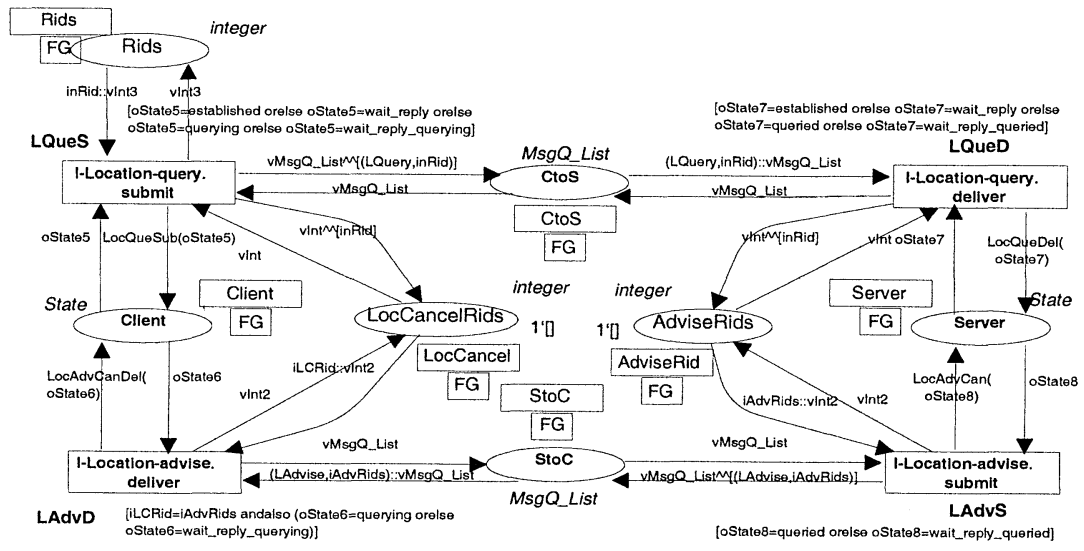

Figure 6. Location Query \& Advise CPN

Figure 7 models the sequence of I-Location-Cancel primitives. The first primitive that can occur in this figure is I-Location-Cancel.submit, given the client is in one of the states (querying, wait_reply_querying) specified in the 
guard and the place LocCancelRids has a Rid (i.e., a location query is outstanding). The transition I-Location-Cancel.deliver can occur next changing server's state to (established or wait_reply) depending on its initial state (i.e., if server is in state queried it is put into state established when ILocation-Cancel.deliver primitive occurs).

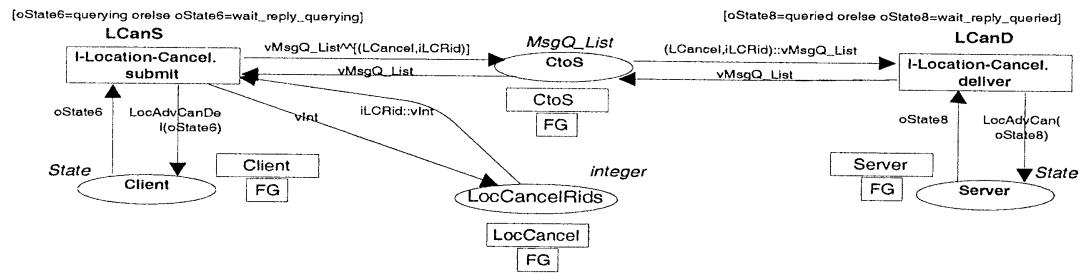

Figure 7. Location Cancel CPN

Figure 8 models the sequence of I-Association-Abort and I-AssociationClose primitives. I-Association-Abort.submit primtive can be issued by the client to close the connection anytime after the connection is established. The server can issue I-Association-Close.submit primitive to close the connection if there are no outstanding requests.

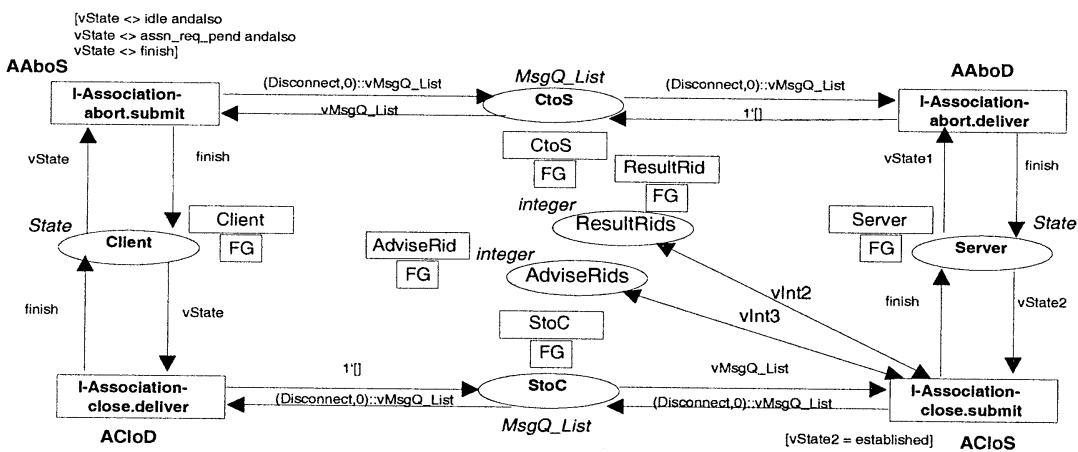

Figure 8. Association Abort \& Close CPN
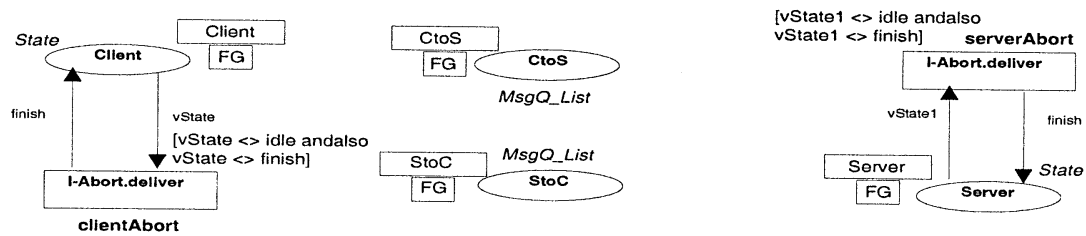

Figure 9. Abort CPN 
Figure 9 models the occurrences of I-Abort primitives. It captures any exceptions that might occur because of an error or malfunction in the underlying layers used by IIOP to deliver its services to ORBs. IAbort.deliver can occur anytime except when the client and the server are in the idle or finish states. The occurrence of I-Abort.deliver puts the client or server into the finish state. The client treats abortive disconnection as an error condition and hence it can retransmit the request after a connection is re-established (not modelled, as we only consider one connection).

\section{Analysis}

The Occurrence Graph (OG) generated by Design/CPN for the IIOP Service CPN contains 1731 nodes and 3864 arcs with 287 terminal states. The graph can be seen as a FSA with the service primitives as the input language. The minimised FSA gives a compact description of the possible sequences of primitives, or service language. This section explains how the minimisation of the OG can remove complexity from the model and presents the IIOP Service language. FSM (AT\&T Research Labs, FSM) is used to produce the minimised FSA. The FSM library is a set of general-purpose software tools available for building, combining, optimizing, searching and comparing finite-state automata.

\subsection{Assumptions}

The model design took into account the analysis techniques that would be applied (e.g., the FSA minimisation). In particular, it was expected that multiple terminal markings would be generated that were only differentiated by the markings of the places connecting the client to server, CtoS and StoC. The model could have used more transitions to remove all tokens from these places once the client has the token, finish. This would ensure only a single terminal marking was generated for this case. However it was left for the FSA minimization to handle the extra markings. FSA minimization merges all terminal markings into one, as a result the models remain free of any "cleanup" transitions.

By treating the OG as a FSA, the introduction of halt states (states that indicate the possible end of a primitive sequence) was necessary. A halt state may or may not lead to other states. As well as all terminal markings being halt states, nodes were defined as halt states if they satisfied either of the following conditions:

- The state of the client and server are both finish

- The state of client is finish and the server is idle 


\subsection{Occurrence Graph Analysis}

The CPN is analysed using 2 request reference identifiers (i.e., fusion place, Rids, has two integers in the list as an initial marking). This is chosen so that both a basic interworking facility transaction and location facility transaction can occur concurrently. It also limits the state space explosion due to many transactions as is evident from Table 4. The first row contains the number of distinct request reference numbers that the client can associate with various basic interworking facility and location facility primitives. The second and third rows have the number of OG nodes and arcs generated for the given number of reference numbers respectively. The fourth row has the status of the generated OG. 'Full' indicates that the complete $\mathrm{OG}$ is generated, while 'Partial' indicates the number of states generated in 300 secs by a Pentium-3, $800 \mathrm{MHz}$ PC with $512 \mathrm{MB}$ of RAM.

Table 4. Statistics for OGs with up to three request reference numbers

\begin{tabular}{|l|l|l|l|}
\hline Number of Reference Ids & 1 & 2 & 3 \\
\hline Nodes Generated & 152 & 1731 & 16701 \\
\hline Arcs Generated & 315 & 3864 & 38192 \\
\hline Status of OG & Full & Full & Partial \\
\hline
\end{tabular}

\subsection{IIOP Service Language}

Even after minimisation, the service language is quite complex and contains 174 nodes. As a result, only a portion of it is presented here in Figure 10. This portion represents successful establishment and release of a connection and possible transactions involving a combination of the basic interworking and location GIF facilities while the connection is established. The arc labels of the FSA use abbreviated names. The abbreviated names are the labels associated with each transition in the CPN. For instance, IAssociation-request.submit is labelled with AReqS in the CPN. Thus the primitive name associated with each arc label in the FSA can be obtained by referring to the appropriate $\mathrm{CPN}$ page.

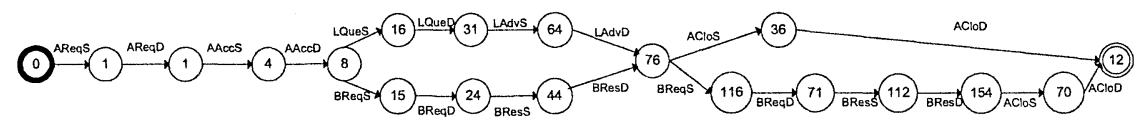

Figure 10 Subset of the IIOP Service Language 
Figure 10 illustrates an expected sequence for a transaction involving, either the Association Facility and Basic Interworking Facility, or, the Association Facility, Location Facility and Basic Interworking Facility. A connection is established by I-Association-request and I-Association-accept primitives by following the sequence of nodes $(0,1,2,4,8)$. A transaction involving only Basic Interworking Facility primitives after the connection is established between the client and the server is observed as node sequence $(8,15,24,44,76)$. The transaction is followed by an orderly shutdown of the connection by a server-initiated close, node sequence $(76,36,12)$. A transaction involving interaction between the location facility and the basic interworking facility is represented by the node sequence $(8,16,31,64,76$, $116,71,112,154)$. This sequence is important as it shows a client may optimise the request by sending a location facility primitive to query the server, to check if it is the right recipient of the request. If the server is the correct recipient of the request, the client may send the basic interworking facility request primitive. The transaction is followed by an orderly shutdown of the connection by a server-initiated close, node sequence (154, $70,12)$. The sequences obtained in the service language, as seen in IIOP Service Language, conform to the expected sequencing specified in the states tables. This increases our confidence that the service specification is correct.

\section{Conclusions}

We combine three of the GIF facilities from ISO/IEC 14752 to define the sequences of primitives for IIOP's service specification, as seen at a local service interface. The sequences of primitives for this combination of GIF facilities is new. The service specification derived here conforms to the high level description of expected services in the CORBA specification. Coloured Petri Nets were then used to formally specify the IIOP service. IIOP's service language (the global set of sequences of service primitives) was determined using reachability analysis and FSA reduction techniques, for a representative combination of transactions, over a single connection.

The modelling of the IIOP service and the generation of the Occurrence Graph (OG) were done using the software tool Design/CPN. The FSA reduction was accomplished using the FSM tool.

Under the assumptions made, the generated IIOP Service Language provides a complete set of service primitive sequences, when considering both interoperating client (requesting) and server (responding) ORBs. This global behaviour is not described in the CORBA specification nor in ISO/IEC 14752. 
The service language can be used as a basis for verifying that IIOP conforms to its service specification. The next step to achieve this is to model the operation of the protocol in detail. This work is in progress and will use the IIOP service language generated in this paper as the yardstick against which IIOP will be verified using a previously developed methodology (Billington et al., 1986).

\section{Acknowledgements}

We would like to thank IBM Global Services Australia for their support to Abhishek Singh during the writing of this paper.

\section{References}

AT\&T Research Labs. FSM Library. http://www.research.att.com/sw/tools/fsm/description.html.

Billington, J. (1983). Abstract specification of the ISO Transport service definition using labelled Numerical Petri nets. In H. Rudin and C. H. West, editors, Protocol Specification, Testing, and Verification, III, pages 173-185. Elsevier Science Publishers, Amsterdam, New York, Oxford.

Billington, J. (1991). FORSEEing Quality Telecommunications Software. Australian Conference on Telecommunications Software, Melbourne.

Billington, J. (1991). Formal Specification of Protocols: Protocol Engineering. Encyclopedia of Microcomputers, vol. 7, Marcel Dekker, Inc. New York and Base, pp. 299-314.

Billington, J., Wilbur-Ham, M.C. and Bearman, M. (1986). Automated Protocol Verification. In Protocol Specification, Testing and Verification, V, pages 59-70, North Holland.

Gordon, S. and Billington, J. (1999). Modelling the WAP Transaction Service using Coloured Petri Nets. In H.-V. Leong, W.-C, Lee, B. Li, and L. Yin, editors, Proc. Of the $1^{\text {st }}$ Int. Conf. On Mobile Data Access, LNCS 1748, pages 109-118, Hong Kong. Springer-Verlag.

International Systems Group Inc. (2001). A Middleware White Paper, Middleware - The Essential component for Enterprise Client/Server Applications.

ISO/IEC 19500-2:2000, Information Technology - Open Distributed Processing -General Inter-ORB Protocol (GIOP)/ Internet Inter-ORB Protocol (IIOP).

ITU-T Recommendation X.210 (1993) | ISO/IEC 10731:1994, Information Technology Open Systems Interconnection - Basic Reference Model - Conventions for the definition of OSI services.

ITU-T Recommendation X.903 (1995) | ISO/IEC 10746-3:1996, Information Technology Open Distributed Processing - Reference Model: Architecture.

ITU-T Recommendation X.931 (1999 E) | ISO/IEC 14752:1999, Information Technology Open Distributed Processing - Protocol support for computational interactions.

Jensen, K. (1997). Coloured Petri Nets. Basic Concepts, Analysis Methods and Practical Use, vol. 1-3. Springer-Verlag, Berlin.

Kristensen, L. M., Christensen, S. and Jensen, K. (1998). The practitioner's guide to Coloured Petri nets. Int J Software Tools for Technology Transfer, 2(2):98-132.

Object Management Group. (2001). The Common Object Request Broker: Architecture and Specification (Revision 2.5). Object Management Group (OMG), Framingham, MA. 